\title{
The use of hemodialysis in hypercalcemic crisis secondary to primary hyperparathyroidism
}

\author{
Catarina Nazário Marouço ${ }^{1}$ (D), Fernando Caeiro ${ }^{1,2}$ iD, Bernardo Marques da Costa $^{1}$ (iD), David Navarro ${ }^{1}$ (iD), Rita Magriço ${ }^{1,2}$, Fernando Nolasco Re $^{1,2}$ \\ ${ }^{1}$ Nephrology Department, Hospital Curry Cabral, Lisboa, Portugal \\ ${ }^{2}$ Nova Medical School, Universidade Nova de Lisboa, Lisboa, Portugal
}

\section{ABSTRACT}

Severe hypercalcemia can be either acute or decompensate from a chronic state to a medical emergency, the hypercalcemic crisis. The presence of symptoms such as altered mental status or potentially fatal ECG abnormalities demand an expeditious decrease in serum calcium levels. Standard medical therapy consists of vigorous volume replacement, calcitonin and, depending on the etiology, bisphosphonates, cinacalcet or glucocorticoids. Hypercalcemic crisis is a rare indication for urgent hemodialysis and is reserved for patients with severe symptoms, ineffective medical therapy or end stage renal disease. The use of hemodialysis in this scenario is not commonly reported. We hereby report a patient who presented with altered mental status and acute kidney injury due to a hypercalcemic crisis secondary to primary hyperparathyroidism. Treatment included urgent hemodialysis to effectively lower calcium levels.

Key-Words: hypercalcemia, primary hyperparathyroidism, hemodialysis

C 2021 Portuguese Journal of Nephrology \& Hypertension. Published by Publicações Ciência \& Vida This is an open access article under the CC BY-NC-ND license (http://creativecommons.org/licenses/by-nc-nd/4.0/).

\section{INTRODUCTION}

Calcium ( $\mathrm{Ca}$ ) is the most abundant ion in the human body and serum ionized calcium ( $\mathrm{Ca} 2+$ ), its active form, is strictly controlled to avoid systemic toxicity, mainly in the neurological, cardiovascular and urinary systems. ${ }^{1}$

Although hypercalcemia is a common electrolyte disorder, the use of urgent hemodialysis for quickly controlling serum calcium levels is infrequent and poorly documented.

This case report describes a patient with severe hypercalcemia due to primary hyperparathyroidism who required hemodialysis to control calcium levels. We will also review the causes, diagnostic approach and treatment of severe hypercalcemia.

\section{CASE REPORT}

A 68-year-old Caucasian male, with no relevant past medical history and a recent diagnosis of a Klatskin tumor, was admitted to the hospital for an elective right hepatectomy and Roux-Y-hepaticojejunostomy. In the postoperative period, he developed an altered mental status characterized by somnolence and lethargy which was assumed to be due to the recent surgery. Laboratory analysis showed a calcium level of $11.5 \mathrm{mg} / \mathrm{dL}$, which had been in the normal range $(8.5 \mathrm{mg} / \mathrm{dL})$ four months before. Intravenous fluids were immediately started. Despite adequate volume expansion and urine output (2500cc/24h), total serum calcium levels increased further to $18.56 \mathrm{mg} / \mathrm{dL}$ (calcium corrected to an albumin level of $2.23 \mathrm{mg} / \mathrm{dL}$ ). His mental status continued to deteriorate and he developed acute kidney injury with serum creatinine $(\mathrm{Scr}) 2.69 \mathrm{mg} / \mathrm{dL}$ which was previously of $0.73 \mathrm{mg} / \mathrm{dL}$. Arterial blood gas showed metabolic alkalemia with a pH of 7.45, pO2 $85 \mathrm{mmHg}$, pCO2 $43 \mathrm{mmHg}$, Ca2+ $3.3 \mathrm{mmol} / \mathrm{L}$, lactate $1.3 \mathrm{mmol} / \mathrm{L}$ and a bicarbonate of $38.7 \mathrm{mEq} / \mathrm{L}$. The electrocardiogram and head-CT were unremarkable.

A diagnosis of hypercalcemic crisis was made and he was started in hemodialysis. The patient was also given $4 \mathrm{mg}$ of zolendronic acid and further volume expansion. After two consecutive daily hemodialysis sessions with a first two-and-a-half hour and a second three-hour length session, low flux, Qb $250 \mathrm{ml} / \mathrm{min}$ and Qd $500 \mathrm{ml} / \mathrm{min}$ with the lowest calcium dialysate available in our center $(1.5 \mathrm{mmol} / \mathrm{L})$, total serum calcium decreased to $12.6 \mathrm{mg} / \mathrm{dL}(\mathrm{Ca} 2+1.7 \mathrm{mmol} / \mathrm{L})$.

Meanwhile, the etiologic investigation showed an elevated intact PTH of $1112.40 \mathrm{pg} / \mathrm{mL}$ accompanied by low Vitamin D $(9.5 \mathrm{ng} / \mathrm{mL})$ and calcitriol $(12.3 \mathrm{ng} / \mathrm{ml})$ levels. Immunofixation did not reveal a monoclonal gammopathy. Neck ultrasound showed a well-delimitated hypoechoic nodule, consistent with a parathyroid adenoma. The diagnosis of primary hyperparathyroidism due to a parathyroid adenoma was made and the patient was started on cinacalcet (30mg once a day).

One week after the two hemodialysis sessions, the patient recovered his previous mental status. Laboratory analysis showed normal renal 
function $(\mathrm{Cr} 0.8 \mathrm{mg} / \mathrm{dL})$ and there was a $50 \%$ reduction of intact PTH (iPTH). No further hemodialysis sessions were required. In fact, due to iatrogenic hypocalcemia secondary to prolonged symbiotic effect of cinacalcet with bisphosphonates, cinacalcet had to be suspended.

The patient was observed by endocrinology and proposed for parathyroidectomy of the affected gland.

\section{DISCUSSION}

Hypercalcemia is a clinical condition defined by a serum total (CaT) or ionized calcium $\left(\mathrm{Ca}^{2+}\right)$ levels above $10.5 \mathrm{mg} / \mathrm{dL}(2.5 \mathrm{mmol} / \mathrm{L})$ and $5.6 \mathrm{mg} / \mathrm{dL}(1.4 \mathrm{mmol} / \mathrm{L}$ ) respectively (Table 1$)$. In this text we will use $\mathrm{mg} / \mathrm{dL}$ when referring to $\mathrm{CaT}$ and $\mathrm{mmol} / \mathrm{L}$ when referring to $\mathrm{Ca}^{2+}$.

\section{Table 1}

Hypercalcemia classification

\begin{tabular}{l|c|c} 
& CaT & Ca2+ \\
\hline Mild hypercalcemia & $10.5-11.9 \mathrm{mg} / \mathrm{dL}$ & $1.4-2 \mathrm{mmol} / \mathrm{L}$ \\
Moderate hypercalcemia & $12-13.9 \mathrm{mg} / \mathrm{dL}$ & $2-2.5 \mathrm{mmol} / \mathrm{L}$ \\
Severe hypercalcemia & $14-16 \mathrm{mg} / \mathrm{dL}$ & $2.5-3 \mathrm{mmol} / \mathrm{L}$
\end{tabular}

Patients with mild hypercalcemia are usually asymptomatic or report nonspecific symptoms. In the vast majority of the cases, symptoms only emerge when serum calcium levels are over $12 \mathrm{mg} / \mathrm{dL}$. The severity of both signs and symptoms depends not only on the absolute value of calcium but also on how rapidly it increases. Chronic hypercalcemia can be fairly asymptomatic, even with moderately elevated calcium levels. Conversely, acute but modest rises in calcium can be associated with severe symptoms. ${ }^{2}$

Hypercalcemia affects most organs. The first symptoms are nonspecific and involve the neuromuscular system. Weakness, malaise, anxiety, depression and cognitive disfunction are well described in the early phases. More severe symptoms like lethargy, confusion, stupor and coma occur with calcium levels $>14 \mathrm{mg} / \mathrm{dL}$ specially in older adults with acute increases. ${ }^{3}$

Gastrointestinal concerns such as anorexia, nausea, vomiting and constipation occur commonly. Peptic ulcer and pancreatitis are less frequent.

Cardiovascular manifestations include electrocardiogram changes with prolonged PR and QRS intervals, short QT interval, T wave flattening or inversion and even ST-elevation sometimes mimicking myocardial infarction. In severe cases, life-threatening supra ventricular and ventricular arrhythmias can occur. ${ }^{4}$

Renal manifestations are numerous and can be divided into two distinct phases. An early phase begins with polyuria and polydipsia secondary to renal salt wasting and nephrogenic diabetes insipidus (NDI). These two phenomena can be explained by two major mechanisms: the first is related to the fact that in the thick ascending limb of the Henle's loop, $\mathrm{Ca} 2+$ transport is linked with the activity of the Na+-K+$2 \mathrm{Cl}$-transport. The stimulation of the $\mathrm{Ca} 2+-$ sensing receptor (CaSR) decreases the permeability to $\mathrm{Ca} 2+$ and consequently the absorption of $\mathrm{Na}+$. This results in a low medullar osmotic gradient and impairs the countercurrent mechanism that is necessary for urinary concentration. The second and probably the most important is the downregulation of aquaporin-2 water channels due to auto-phagic degradation resulting in an incapacity of absorbing free water in the collecting tubule. ${ }^{5}$

Without adequate fluid replacement, this will result in hypovolemia leading to a second phase of pre-renal acute kidney injury. Volume depletion increases water, $\mathrm{NaCl}$, bicarbonate ( $\mathrm{HCO} 3$ ) and (by convection) calcium reabsorption in the proximal tubule. The resulting metabolic alkalemia activates the $\mathrm{pH}$ dependent calcium receptor present in the distal tubule exacerbating even more this state of hypercalcemia. Chronic hypercalcemia can lead to tubulointerstitial lesions and cortical nephrocalcinosis.

The initial and most important goal of laboratory evaluation is to differentiate parathyroid hormone (PTH)-mediated hypercalcemia (primary hyperparathyroidism and familial hyperparathyroid syndromes) from non-PTH mediated hypercalcemia (malignant tumors, vitamin $\mathrm{D}$ intoxication, granulomatous disease). Thus, once hypercalcemia is confirmed, the next step is to determine serum intact PTH (Figure 1). Primary hyperparathyroidism is the most common cause of hypercalcemia and benign parathyroid adenomas constitute more than $80 \%$ of these cases. ${ }^{2}$ Humoral hypercalcemia of malignancy is the second most common etiology. ${ }^{6}$ Excessive bone resorption by direct invasion (metastases) or by producing osteoclastic factors, mainly PTHrP (PTH-related peptide), are the major mechanisms in squamous cell carcinoma (lung, head and neck), renal, bladder, breasts or ovarian carcinomas. Together, malignant tumors and primary hyperparathyroidism are responsible for more than $90 \%$ of hypercalcemia cases. The remaining $10 \%$ are due to miscellaneous causes: excessive Vitamin D or calcitriol consumption; granulomatous diseases like sarcoidosis and lymphomas due to uncontrolled extra renal production of calcitriol. Tuberculosis, leprosy and berylliosis are rare causes of hypercalcemia probably through the same mechanism (calcitriol production by macrophages). The presence of low PTH, PTHrp and low or normal Vitamin D metabolites suggest other causes like multiple myeloma, especially in the elderly, thyrotoxicosis, immobilization, Vitamin A toxicity or excessive calcium intake, as in milk alkali syndrome. ${ }^{7}$

Although hypercalcemia is common, hypercalcemic crisis is an unusual endocrine medical emergency. There are few cases described in the literature and due to its relative rarity, hypercalcemic crisis has no standard definition. However, it is commonly accepted as an albumincorrected serum calcium level greater than $14 \mathrm{mg} / \mathrm{dL}$ associated with the presence of multi-organ disfunction (neurological, cardiovascular, gastrointestinal and/or renal). The diagnosis should also be considered in severely symptomatic patients despite lower calcium levels. ${ }^{4}$

The majority of hypercalcemia crises are caused by primary hyperparathyroidism and originate from an acute decompensation of previous hypercalcemia. Hypercalcemia from malignancy or other causes of hypercalcemia rarely increases to such high levels. ${ }^{4}$

Once the diagnosis of hypercalcemic crisis is established, two basic strategies should be undertaken: urgent lowering of calcium serum levels, given the high mortality if not treated promptly, and etiologic investigation. 


\section{Figure 1}

A pratical approach to hypercalcemia - adapted from Up To Date "Diagnostic approach to hypercalcemia"

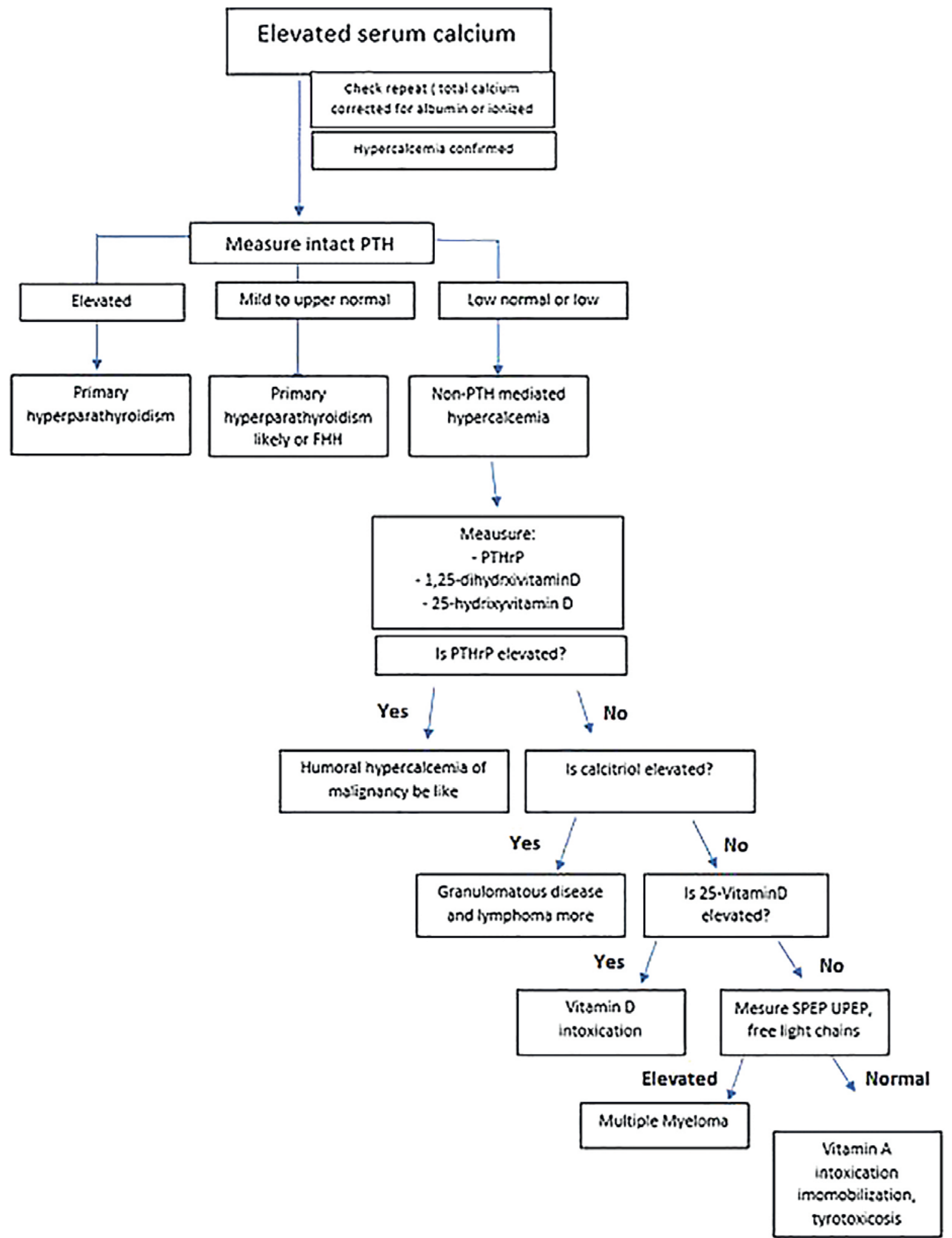


As explained above, individuals with hypercalcemic crisis are hypovolemic and sodium depleted. For this reason, the most important factor in the initial approach consists of adequate volume expansion. This increases filtered calcium and decreases calcium re-absorption in the proximal tubule, thereby promoting calciuresis. A practical approach consists of 3 to $4 \mathrm{~L}$ of saline infusion for 24 hours, followed by 2 to $3 \mathrm{~L}$ until adequate urine output is established ( $2 \mathrm{~L} /$ day). Intravenous volume expansion per se reduces hypercalcemia in most patients (with expected reductions from 1.6 to $2.4 \mathrm{mg} / \mathrm{dL}$ ) but will be insufficient to restore normocalcemia in individuals with higher serum calcium levels. ${ }^{8}$

The second immediate measure to apply is calcitonin administration. It has the most rapid onset of action of all available drugs. In combination with volume expansion it's effective in lowering serum calcium within 12 to 24 hours by reducing bone resorption and promoting calciuresis as well. Its use is limited by the rapid development of tachyphylaxis and therapeutic tolerance. Unfortunately, it is also unavailable in many centers. ${ }^{9}$

Bisphosphonate therapy is particularly effective in hypercalcemia of malignancy and is considered first-line treatment for hypercalcemia of any etiology. They act by blocking osteoclast-mediated bone reabsorption via osteoclast apoptosis and are one of the most effective therapies. The onset of action is 48 to 72 hours to take effect and overall duration is $28-30$ days. Zoledronate has been reported to be more efficient than pamidronate and can be infused over 15 minutes. The combination of intravenous fluid replacement, calcitonin (when available) and bisphosphonates is considered the standard medical therapy for all patients with severe hypercalcemia and should be started as soon as possible. ${ }^{10}$

Loop diuretics are often combined with isotonic saline infusion, allowing an increase in calciuresis. They should only be administered after adequate volume repletion, given the risk of circulatory collapse. Individuals with limited cardiac reserve particularly benefit from this combination. Thiazide diuretics are contraindicated as they enhance calcium reabsorption in the distal nephron.

Regarding dialysis, there is neither high-quality evidence nor a broad consensus on its use. Reports of it in the literature are scarce. There is some consensus that hemodialysis should be considered the preferential treatment in patients in whom the promotion of calciuresis is not viable (i.e. severe acute kidney injury, end stage kidney disease, congestive heart failure etc). ${ }^{11}$

Hemodialysis is also effective when hypercalcemia is refractory to standard treatment or when emergent reduction is needed while awaiting more definitive therapy. Hypercalcemic crisis with neurological or cardiovascular involvement is an emergency and hemodialysis is considered first-line therapy in combination with medical standard therapy. ${ }^{11}$

Another issue in debate is what dialysate calcium concentration should be used and there is no consensus on this aspect either. Many institutions choose calcium-free dialysate during hemodialysis of patients with hypercalcemic crisis. However, the use of low calcium dialysate $<1$ to $1.5 \mathrm{mmol} / \mathrm{l}$ seem to be equally effective. Moreover, as calcium ions have an important role in cardiac iono and cronotropism, excessive diffusion may lead to hemodynamic instability. ${ }^{12}$
Due to the phosphaturic effect of hyperparathyroidism, these patients are usually hypophosphatemic. Some authors suggest the use of phosphorous-enriched dialysate while other prefer intravenous phosphorous correction. Polyuria can also lead to hypokalemia that should be corrected as well.

Our patient presented with a total calcium level of $18.56 \mathrm{mg} / \mathrm{dL}$ and severe progressive neurological involvement. The diagnosis of hypercalcemic crisis was made and emergent treatment was necessary. Considering that the effect of IV fluid expansion is modest and the patient already had an adequate urine output, that calcitonin is unavailable in our institution and that the effect of bisphosphonates has a significant delay, a decision to start hemodialysis was made.

The next step after urgent calcium lowering has been achieved consists of applying specific treatment according to the etiology of hypercalcemia. Cinacalcet, a calcimimetic agent, is approved as firstline therapy in hypercalcemia secondary to parathyroid carcinoma and in secondary hyperparathyroidism due to end stage renal disease. In patients with primary hyperparathyroidism of any cause and severe symptomatic hypercalcemia who are waiting for or have a contraindication to parathyroidectomy, the use of cinacalcet is indicated as well $(30 \mathrm{mg}$ bid with PTH and calcium values reassessment after one week). Parathyroidectomy is the definitive treatment of primary hyperparathyroidism secondary to parathyroid adenoma.

It should also be noted, as in our case, that there appears to be a higher incidence of primary hyperparathyroidism in patients with malignancy and vice versa. In a scenario where both PTHrp and PTH are elevated, co-existence of primary hyperparathyroidism and hypercalcemia of malignancy is also possible. PTHrp is not available in our center and due to the fact that our patient improved consistently, measurement of PTHrp was not performed.

\section{CONCLUSION}

Hypercalcemic crisis is an unusual life-threatening medical emergency associated with high mortality if not treated early and promptly. It must be managed immediately. The vast majority of the cases are secondary to primary hyperparathyroidism and usually result from decompensation of previous chronic hypercalcemia.

In this situation, as emergent reduction of calcium is warranted, hemodialysis should be considered first-line therapy in addition to standard medical therapy. At the same time an etiologic investigation should be initiated in order to institute a directed and definitive therapy.

Disclosure of potential conflicts of interest: none declared.

\section{References}

1. Sakuta A, lijima T, Domeki N, et al. The etiology, clinical features and management of hypercalcemia and its crisis. Dokkyo J Med 2012; 39: 45-55

2. Ahmad S, Kuraganti G, Steenkamp D. Hypercalcemic crisis: A clinical review. Am J Med 2015; 128 : 239-245

3. Wang C, Guyton S. Hyperparathyroid crisis: clinical and pathological studies of 14 patients. Ann Surg 1979; 190(6): 78-82 
4. Ziegler R. Hypercalcemic Crisis. J Am Soc Nephrol 2001; 12: S3-S9.

5. Feehally John, Floege Jurgen, Tonelli Marcello, Johnson Richard. Comprehensive Clinical Nephrology. 6th Edition. Elsevier, 2018.

6. Mundy G, Guise T. Hypercalcemia of Malignancy. Am J Med 1997; 103:103-105

7. Lafferty, F. Differential diagnosis of hypercalcemia. J Bone Miner Res 1991; 6: 51-59

8. Massry S, Coburn JW, Chapman L, Kleeman C. Role of serum calcium, parathyroid hormone, and $\mathrm{NaCL}$ infusion on renal Ca and Na clearances. Am J Physiol 1968; 214: 1403-9

9. Thiebaud D, Jacquet AF, Burckhardt P. Fast and effective treatment of malignant hypercalcemia. Combination of suppositories of calcitonin and a single infusion of 3-amino 1- hydroxypropylidene1-bisphosphonate. Arch Intern Med 1990; 150:2125-8

10. Major P, Lortholary A, Hon J, et al. Zoledronic acid is superior to pamidronate in the treatment of hypercalcemia of malignancy: a pooled analysis of two randomized, controlled clinical trials. J Clin Oncol 2001; 19: 558-67

11. Loh HH, Mohd Noor N. The Use of Hemodialysis in Refractory Hypercalcemia Secondary to Parathyroid Carcinoma. Case Rep Crit Care 2014, 2014:140906.

12. Leehey DJ, Todd S. Correction of hypercalcemia and hypophosphatemia by hemodialysis using a conventional, calcium-containing dialysis solution enriched with phosphorus. Am J KidneyDiseases 1997; 29:288- 290

\section{ORCID}

Catarina Nazário Marouço (iD) 0000-0003-1861-1072

Fernando Caeiro (iD) 0000-0002-7294-1630

Bernardo Marques da Costa (iD) 0000-0002-7639-5390

David Navarro (iD 0000-0002-1977-794X

\section{Correspondence to:}

Catarina Marouço, MD

Nephrology Department, Hospital Curry Cabral

Centro Hospitalar Lisboa Central, Lisboa, Portugal

Email: catarina.marouco@gmail.com 